\title{
A newly discovered accreting pulsar in Terzan 5
}

\author{
A. Papitto ${ }^{(a, b) *}$, S. Motta ${ }^{(c)}$, A. Riggio ${ }^{(b)}$, A. D’Aì ${ }^{(d)}$, T. Di Salvo ${ }^{(d)}$, T. Belloni ${ }^{(c)}$, \\ L. Burderi $(a)$, R. laria $(d) *$ \\ ${ }^{(a)}$ Dipartimento di Fisica, Università di Cagliari, ${ }^{(b)}$ INAF, Osservatorio Astronomico di \\ Cagliari, ${ }^{(c)}$ INAF - Osservatorio Astronomico di Brera, ${ }^{(d)}$ Dipartimento di Fisica, Università \\ di Palermo \\ E-mail: apapittodoa-cagliari.inaf.it
}

The $11 \mathrm{~Hz}$ accreting pulsar, IGR J17480-2446, was recently discovered in the globular cluster Terzan 5. The analisys of the Doppler shifts induced by the orbital motion reveals how the neutron star belongs to a $\sim 21.3 \mathrm{hr}$ binary system with a companion star of $0.4-1.5 \mathrm{M}_{\odot}$. The X-ray pulsar spins up while accreting at an average rate of $1.48(2) \times 10^{-12} \mathrm{~Hz} \mathrm{~s}^{-1}$, in agreement with the accretion of disc matter angular momentum given the observed luminosity. From the presence of pulsations at different accretion rates we constrain the magnetic field to lie within $\sim 2 \times 10^{8}$ and $\sim 2 \times 10^{10} \mathrm{G}$. From this estimate, the value of the spin period and of the observed spin-up rate, we associate this source with the still poorly sampled population of slow, mildly recycled, accreting pulsars.

The pulse profile is generally modelled by using two harmonic components, and a timing analysis performed on the pulse phases shows how the second harmonic is much less affected by timing noise than the first one. The temporal evolution of the pulse phases computed over the second harmonic is also much more in agreement with the expected dependence of the spin-up rate on the accretion luminosity. The assumption that this harmonic component is a good tracer of the neutron star spin frequency opens the possibility of a dynamical measure of the accretion torque exerted on this neutron star during accretion.

Fast $X$-ray timing and spectroscopy at extreme count rates

February 7-11, 2011

Champéry, Switzerland

\footnotetext{
* Speaker.
} 


\section{Introduction.}

To perform a temporal analysis of the X-ray coherent signal emitted by an accreting pulsar is one of the foremost techniques to investigate the dynamical interaction between the rotating neutron star (NS hereafter) and the accretion disc surrounding it. Besides giving an insight into the orbital system the NS belongs to, the measure of the NS spin frequency variation possibly allows an estimates of the torque it experiences as mass is accreted.

A very appealing case in this regard is that of the recently discovered $11 \mathrm{~Hz}$ pulsar, IGR J17480-2446, belonging to the globular cluster Terzan 5. The relatively frequent stellar encounters taking place in a globular cluster greatly enhance the formation of binary systems hosting a NS. The very large density of Terzan 5 when compared to other clusters [1] therefore translates in this cluster having a large number (33) of rotation powered millisecond pulsars [2]. According to the recycling scenario (see, e.g., [3]) such quickly-rotating pulsars are the end-product of a long lasting phase of accretion of matter and of the relative angular momentum by the NS in a low-mass X-ray binary (LMXB). The presence of 28 discrete X-ray sources in Terzan 5, a substantial number of which can be identified as quiescent LMXB [4], further points in favour of an evolutionary link between the populations of rotation-powered millisecond pulsars and LMXB in this cluster.

The outburst of a new transient X-ray source in Terzan 5 was first observed on 2010 October 10 by INTEGRAL [5] and the newly discovered source termed as IGR J17480-2446 (J17480 in the following). Subsequent Rossi X-ray Timing Explorer (RXTE) observations detected a coherent signal at a frequency of $11 \mathrm{~Hz}$ making this source a newly discovered accreting pulsar [6]. Further, the source showed also a very large number $(\sim 400)$ of X-ray bursts during its outburst, with a recurrence time as short as few minutes and highly dependent on the source mass accretion rate. An analysis of the burst energetics relative to the persistent emission strongly indicates how these bursts are of thermonuclear origin [7, 8,9], while still very peculiar since such a high bursting rate was never observed from a type-I burster. Pulsations at $11 \mathrm{~Hz}$ were observed also during bursts, while burst oscillations have never been observed before from NS rotating at periods exceeding a few ms (see S. Motta and Y. Cavecchi poster contributions at this conference). Here we report about the properties of the coherent signal shown by $\mathrm{J} 17480$ during its non-bursting emission, presenting also preliminary results on its frequency evolution from the modelling of the pulse phases.

\section{Observations and results.}

\subsection{Light curve and spectrum.}

The results presented here are based on the analysis of the observations performed by RXTE and Swift between 2010, October 10 and November 19 (MJD 55479 and 55519, respectively). To avoid contamination of the burst emission we discard $10 \mathrm{~s}$ prior the onset of each of the bursts that could be significantly detected above the continuum emission, and a variable interval of length between 50 and $160 \mathrm{~s}$ after, depending on the burst decay timescale. The source light curve is plotted in the left panel of Fig. 1. The X-ray luminosity increases during the first $\sim 8 \mathrm{~d}$ of the outburst, attaining a peak value of $9.2(1) \times 10^{37} \mathrm{~d}_{5.9}^{2} \mathrm{erg} \mathrm{s}^{-1}$, where a distance estimate of 5.9(5) kpc was considered [1]. During the outburst decay the source became unobservable on MJD 55519.1 due to a solar occultation. After the considered region of the sky became again observable on MJD 

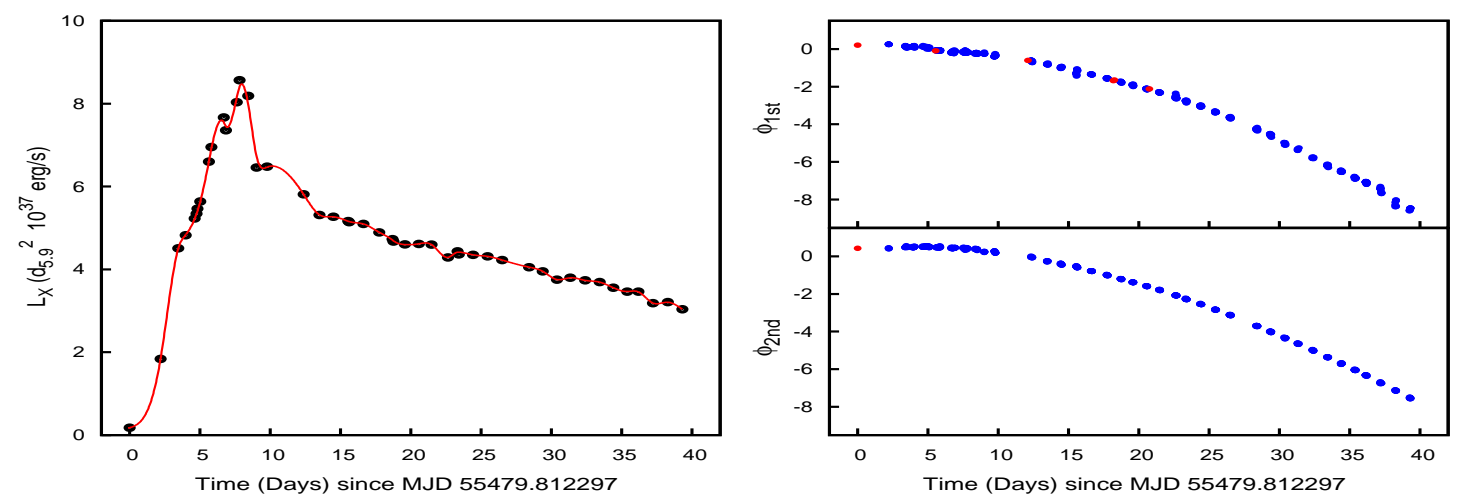

Figure 1: (Left panel) Light curve of the J17480 outburst. Bolometric estimates of the unabsorbed flux were obtained extrapolating the best-fitting models of the spectra observed in the $0.5-10 \mathrm{keV}$ (XRT onboard Swift) and 3-30 keV (PCA on board RXTE) to a 0.1-100 keV band. (Right panel) Pulse phases computed on the first (top) and on the second (bottom) harmonic. Blue points represent $R X T E$ observation, while red ones correspond to Swift pointings.

55584.6 (2011, January 23.6), further RXTE observations detected no excess with respect to the background indicating how the J17480 outburst ended during the period of non observability.

The $0.5-10 \mathrm{keV}$ X-ray spectrum observed by Swift during the first outburst observation available can be well modelled by an absorbed blackbody [12]. Extrapolating such component over a $0.1-100 \mathrm{keV}$ energy band leads to an estimate of the luminosity of $L_{\min }=0.20(2) \times 10^{37} \mathrm{~d}_{5.9}^{2}$ erg $\mathrm{s}^{-1}$. The 3-30 keV spectra observed by the PCA aboard RXTE during the subsequent observations can be well modelled by a thermal Comptonized component (such as nthcomp) and a Gaussian emission line centred at $\sim 6.5 \mathrm{keV}$. A significant softening of the spectrum takes place between MJD 55482.0 and 55483.3 (between days 2.2 and 3.5 according to the temporal scale used in Fig. 1 ), as the temperature of the Comptonizing cloud decreases from $\sim 7 \mathrm{keV}$ to $\sim 3 \mathrm{keV}$. During all the rest of the outburst the spectral parameters of the source remain quite constant, as a model describing the Comptonization of $\sim 1 \mathrm{keV}$ seed photons off a relatively cold $\left(\mathrm{kT}_{e} \sim 3 \mathrm{keV}\right)$ and optically thick $(\tau \sim 9)$ medium is the only component needed to fit the spectral continuum observed by RXTE. The luminosity values quoted above, as well as those plotted in the left panel of Fig 1, were obtained by extrapolating the observed fluxes over the $0.1-100 \mathrm{keV}$ band.

\subsection{The binary system.}

To analyse the properties of the coherent pulsations shown by the source we consider data taken by the PCA on-board RXTE in Event and Good Xenon mode (with temporal resolution of 122 and $1 \mu \mathrm{s}$, respectively), and by the XRT on-board Swift in Windowed timing mode (temporal resolution of $1.7 \mathrm{~ms}$ ). Photon arrival times were preliminarily reported to the Solar System barycentre using the Chandra estimate of the source position [10]. Standard techniques of analysis of a coherent signal were used to estimate the orbital parameters of the binary system (see [11] for details). The J17480 orbit has a period of 21.2745(1) hr and a projected semi-major axis of 2.4971(1) lt-s ${ }^{1}$. Such values translate into a mass function as large as $f\left(M_{2} ; M_{1}, i\right)=0.021269(9)$

\footnotetext{
${ }^{1}$ Slight differences, still within the estimated uncertainties, between the values presented here and those published
} 
$\mathrm{M}_{\odot}$, thus placing a lower limit on the companion mass of $0.41 \mathrm{M}_{\odot}$ (for a NS mass of $1.4 \mathrm{M}_{\odot}$ and an inclination of $90^{\circ}$ ). An upper limit of $2.75 \mathrm{M}_{\odot}$ is obtained by assuming that the companion star fills its Roche Lobe while transferring mass and follows a mass-radius relation $R_{2} \propto M_{2}$. Such limit exceeds the maximum mass expected for a main sequence star belonging to the populations of Terzan 5 (1.5 $\mathrm{M}_{\odot}$ if the star belongs to a population $4 \mathrm{Gyr}$ old, D' Antona, private communication). It is then possible to conclude that the companion of $\mathrm{J} 17480$ has a mass in the range $0.4-1.5 \mathrm{M}_{\odot}$, indicating a main sequence or a slightly evolved star. J17480 is thus the first accreting pulsar to be discovered in an intermediate-to-low mass X-ray binary with a spin period between 10 and 100 $\mathrm{ms}$, and possibly represents a mildly-recycled pulsar.

\subsection{The pulse profile.}

Two harmonic components are generally sufficient to model the pulse profiles obtained by folding the light curves around the best estimate of the spin frequency. The pulsed fraction is observed to greatly vary between MJD 55482.0 and 55483.3, simultaneously to the softening observed in the RXTE spectrum. While the fractional amplitude of the first harmonic component before MJD 55483.2 is $\sim 25 \%$ both during Swift and RXTE observations, it decreases to values around $2 \%$ during the rest of the outburst. A similar variation is not observed for the second harmonic component, the fractional amplitude of which remains roughly constant around $\sim 1 \%$. Since the second harmonic has a strength often comparable and sometimes slightly larger than the first one, the inclination and the misalignment between the magnetic and spin axes are probably such that both the hotspots on the NS surface are seen, at least for a fraction of the rotational phases.

The detection of pulsations throughout the observations considered (except for some of the Swift pointings during which the statistics accumulated was too low) allows to place constraints on the NS magnetic field strength. For accretion to proceed and pulsations to be observed, the inner disc radius, $R_{i n}$, has to lie between the NS radius and the co-rotation radius, that is the distance from the NS where the velocity of the magnetosphere equals the Keplerian velocity of the disc matter $\left(R_{C}=338 \mathrm{~m}_{1.4}^{1 / 3} \mathrm{~km}\right.$ for $\mathrm{J} 17480$, where $\mathrm{m}_{1.4}$ is the NS mass in units of $\left.1.4 \mathrm{M}_{\odot}\right)$. The inner disc radius can be expressed in terms of the pressure equilibrium between the disc and the magnetosphere, $R_{\text {in }} \simeq 160 \mathrm{~m}_{1.4}^{1 / 7} \mathrm{R}_{6}^{-2 / 7} \mathrm{~L}_{37}^{-2 / 7} \mu_{28}^{4 / 7} \mathrm{~km}$, where $\mathrm{R}_{6}$ is the NS radius in units of $10 \mathrm{~km}$, $\mathrm{L}_{37}$ the accretion luminosity in units of $10^{37} \mathrm{erg} / \mathrm{s}$ and $\mu_{28}$ the magnetic dipole moment in units of $10^{28} \mathrm{G} \mathrm{cm}^{3}$. Considering the estimates of maximum and minimum luminosity given above, and imposing $\mathrm{R}_{N S} \leq \mathrm{R}_{\text {in }} \leq \mathrm{R}_{C}$, estimates of the dipole magnetic moment in the range between $\sim 2 \times 10^{26}$ $\mathrm{d}_{5.9}$ and $\sim 1.7 \times 10^{28} \mathrm{~d}_{5.9} \mathrm{G} \mathrm{cm}^{3}$ are obtained. These translate to a magnetic flux density at the NS surface between $\sim 2 \times 10^{8} \mathrm{~d}_{5.9}$ and $\sim 1.7 \times 10^{10} \mathrm{~d}_{5.9} \mathrm{G}$.

\subsection{The spin evolution.}

We plot in the right panel of Fig. 1 the temporal evolution of the phases computed over the first (top panel) and the second harmonic (bottom panel) used to model the pulse profile of J17480. The need for at least a quadratic component to model the temporal evolution of both harmonics phases strongly indicates a spin frequency increase during the outburst. By modelling such evolution with

in [11] owe to the longer time interval covered by the dataset considered here. 

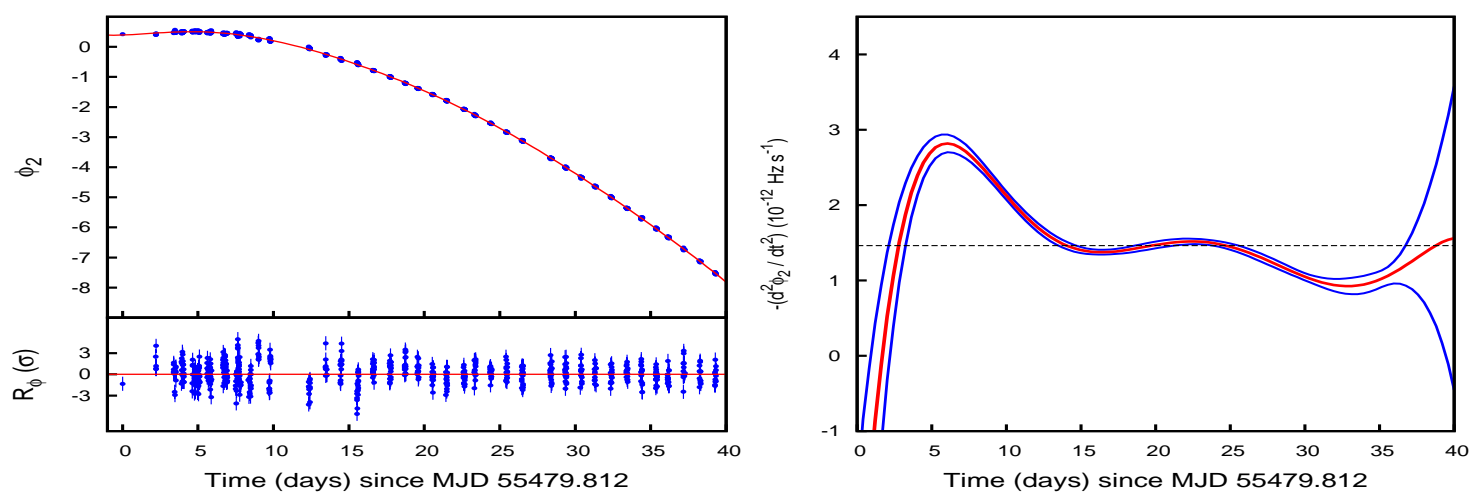

Figure 2: (Left panel) Phases computed on the second harmonic component. The red solid line is the best fitting 8-th order polynomial (top panel), while residuals in units of $\sigma$ with respect to this model are shown in the bottom panel. (Right panel) Second derivative of the polynomial expansion that best fits the second harmonic phases (red line). Confidence intervals at a $1 \sigma$ level are shown as blue lines, and the average value of the spin frequency derivative as a dashed horizontal line.

a constant spin frequency derivative model (that is with a parabolic function) an estimate of the average derivative as $\langle\dot{v}\rangle=1.48(2) \times 10^{-12} \mathrm{~Hz} \mathrm{~s}^{-1}$ is obtained.

The most probable interpretation of the observed spin evolution is in terms of the accretion of the angular momentum of the in-falling matter. Assuming that the NS simply gains the Keplerian angular momentum of the disc matter at the inner disc radius, $\mathrm{R}_{i n}$, and that the mass accretion rate is well tracked by the X-ray luminosity, one has

$$
\dot{v} \simeq 1.5 \times 10^{-12}\left(L_{37} / 5\right)\left(R_{\text {in }} / 70 \mathrm{~km}\right)^{1 / 2} I_{45}^{-1} R_{6} m_{1.4} \mathrm{~Hz} \mathrm{~s}^{-1},
$$

where $I_{45}$ is the NS moment of inertia in units of $10^{45} \mathrm{~g} \mathrm{~cm}^{2}$. The measured average spin up rate is of the same order of the acceleration imparted to the NS by accretion of mass at the observed rate, if the disc is truncated at a distance from the NS compatible with accretion to proceed.

Nevertheless, the evolution of the phases measured over both harmonic components is far from being adequately described by a constant spin up rate. This is well summarised by the large values of the $\chi_{r}^{2}$ obtained when fitting the phases with such a model $\left(\chi_{r}^{2} \simeq 10\right.$ over 676 degrees of freedom for the second harmonic phases, $\chi_{r}^{2} \simeq 21$ over 766 for the first harmonic). Besides showing a milder effect of timing noise on the phases computed over the second harmonic component rather than on the first one (a phenomenon already observed in several cases of accreting pulsars, see, e.g., [13]), these large values indicate the need of a higher order polynomial to model the long term phase behaviour, possibly in accordance with the temporal evolution of the accretion luminosity. To show this, the phases of both harmonic components were fitted with a series of polynomials of increasing order, chosen in order for them to be orthogonal on the given interval. The choice of such a base avoids any correlation between polynomials of different orders in the fit.

Preliminary results show how an 8-th order polynomial expansion is adequate to fit the second harmonic phases, since no significant improvement is obtained by adding highest order terms (see left panel of Fig. 2). The quality of the modelling greatly improves with respect to the simple constant spin-up model, as it is shown by the value of the $\chi_{r}^{2}$ obtained (2.0 over 673 d.o.f.). Further, the second harmonic keeps proving itself as the most stable component since a fit with a polynomial 
of the same order of the first harmonic phases gives $\chi_{r}^{2} \simeq 6.1$ (over 760 d.o.f.). The shape of the best-fitting polynomial curve also better agrees with the expectation of a spin-up rate dependence on the mass accretion rate $\left(\dot{v} \propto \dot{M} R_{i n}^{1 / 2}(\dot{M}) \approx \dot{M}\right)$. To show this, we plot in the right panel of Fig. 2 the opposite of the second derivative of the 8-th order polynomial expansion that best-fits the second harmonic phases, which equals the spin-up rate under the hypothesis that the evolution of the phases computed over the second harmonic closely tracks that of the NS rotation frequency. The trend qualitatively resembles that of the X-ray light curve (left panel of Fig. 1), while the second derivative of the polynomial that best fits the first harmonic phases shows irregular swings probably caused by timing noise. A maximum spin up rate of $2.8(1) \times 10^{-12} \mathrm{~Hz} \mathrm{~s}^{-1}$ is reached around MJD 55485, within less than $2 \mathrm{~d}$ from the attainment of the peak X-ray luminosity. Considering the peak value of the luminosity, $L_{X}^{\max }$, and using Eq. 2.1 to relate the spin-up rate to the accretion luminosity, one obtains an estimate of the inner disc radius as large as $R_{\text {in }}=70 \pm 20 \mathrm{~km}$, where the uncertainty on the distance to Terzan 5 was taken into account. Such an estimate indicates a slightly larger inner disc radius than that obtained if the $815 \mathrm{~Hz}$ QPO detected from J17480 while the source attained its peak flux is assumed to originate at the inner disc boundary ( $\lesssim 20 \mathrm{~km},[14]$ ). This analysis shows how the second harmonic component seems a much better tracer of the spin frequency evolution of J17480 than the first harmonic component. Despite being still preliminary, fitting the second harmonic phases with a model that directly relates the expected torque to the $\mathrm{X}$-ray luminosity gives similar results to those given here, and will be presented in its full details elsewhere (Papitto et al., in prep.).

\section{Acknowledgments}

AP acknowledges the support of the operating program of Regione Sardegna (ESF 20072013), L.R.7/2007, "Promotion of scientific research and technological innovation in Sardinia".

\section{References}

[1] B. Lanzoni, F. R. Ferraro, E. Dalessandro, et al., ApJ 2010 (653) 717 [arXiv:1005.2847]

[2] S. M. Ransom, J. W. T. Hessels, I. H. Stairs, et al., Science 1995 (307) 892 [arXiv:0501230v1]

[3] D. Bhattacharya \& E. P. J. van den Heuvel, Phys.Rep. 1991 (203) 1

[4] C. Heinke, R. Wijnands, H. N. Cohn, et al., ApJ 2006 (651) 1098 [arXiv:astro-ph/0606253]

[5] P. Bordas, E. Kuulkers, J.Alfonso-Garzon, et al., ATel 2010 (2919) 1

[6] S. E. Strohmayer and C. B. Markwardt, ATel 2010 (2929) 1

[7] S. Motta, A. D’Aì, A. Papitto, et al., MNRAS 2011 (414) 1508

[8] M. Chakraborty \& S. Bhattacharyya, ApJ 2011 (730) L23

[9] M. Linares, D. Chakrabarty, M. van der Klis, ApJ 2011 (733) L17

[10] D. Pooley, J. Homan, C. Heinke, et al., ATel 2010 (2974) 1

[11] A. Papitto, A. D’ Aì, S. Motta, et al., A\&A 2011 (526) L3 [arXiv:1010.4793]

[12] E. Bozzo, C. Ferrigno, P. Bordas, ATel 2010 (2922) 1 
[13] L. Burderi, T. Di Salvo, M. T. Menna, ApJ 2006 (653) L133 [arXiv:astro-ph/0612093]

[14] D. Altamirano, J. Homan, M. Linares, et al., ATel 2010 (2952) 1 